\title{
Softly broken lepton numbers: an approach to maximal neutrino mixing
}

\author{
Luís Lavoura* \\ Universidade Técnica de Lisboa \\ Centro de Física das Interacções Fundamentais \\ Instituto Superior Técnico, P-1049-001 Lisboa, Portugal \\ E-mail: 'balio@cfif.ist.utl.pt,
}

ABSTRACT: In the extension of the standard model with three right-handed neutrinos and the seesaw mechanism, we assume that the generation lepton numbers are separately conserved in the Yukawa couplings, and that they are softly broken by the Majorana mass matrix of the neutrino singlets. Deviations from family-lepton-number conservation are then calculable. In this framework, it is possible to introduce symmetries such that either maximal atmospheric-neutrino mixing, or maximal solar-neutrino mixing, or both simultaneously, are achieved naturally.

I am going to report on work which has been done in collaboration with Walter Grimus,

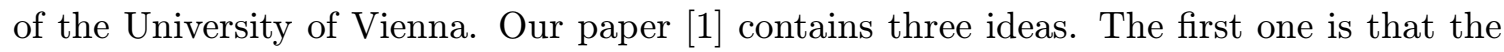
three individual family lepton numbers $L_{e}, L_{\mu}$, and $L_{\tau}$ may constitute symmetries of the lagrangian which are broken only softly by the Majorana masses of right-handed neutrinos. From this idea it follows that the soft breaking of the lepton numbers occurs through superhigh (of order $10^{11} \mathrm{GeV}$ or so) mass terms, instead of proceeding through small masses, as is usually argued in connection with the soft breaking of symmetries. We claim that this idea makes sense because, due to the soft breaking of the family lepton numbers, the renormalization-group equations do not induce family-lepton-number-violating Yukawa couplings; all lepton-number-breaking effects turn out to be calculable (finite) and, indeed, suppressed. The second idea consists of a model which, within the framework described above, leads to maximal atmospheric-neutrino mixing. The third idea (which is separate and distinct from the second one) is a model for maximal solar-neutrino mixing. At this point, I want to stress that when we are talking of models we thereby understand theories with well-defined particle contents, symmetries, and mechanisms of symmetry breaking. Our models are not Ansätze, "textures", or "patterns", rather they are self-contained theories, consistent within a quantum-field-theoretical framework.

\footnotetext{
${ }^{*}$ Speaker.
} 
In order to set the stage, I start with the weak leptonic current:

$$
j^{\alpha}=(\bar{e}, \bar{\mu}, \bar{\tau}) \gamma^{\alpha} \gamma_{L}\left(\begin{array}{ccc}
U_{e 1} & U_{e 2} & U_{e 3} \\
U_{\mu 1} & U_{\mu 2} & U_{\mu 3} \\
U_{\tau 1} & U_{\tau 2} & U_{\tau 3}
\end{array}\right)\left(\begin{array}{c}
\nu_{1} \\
\nu_{2} \\
\nu_{3}
\end{array}\right)
$$

where the matrix $U$ is the lepton mixing matrix. We know that the mass-squared differences among the three (light) neutrinos have distinct orders of magnitude:

$$
\Delta m_{\text {atm }}^{2}=\left|m_{3}^{2}-m_{2}^{2}\right| \gg\left|m_{2}^{2}-m_{1}^{2}\right|=\Delta m_{\odot}^{2} .
$$

We also know, from the CHOOZ and Super-Kamiokande experiments, that $\left|U_{e 3}\right| \ll 1$. Finally, as we have already learned in this parallel session, experiment indicates that atmospheric-neutrino mixing is maximal, $\left|U_{\mu 3}\right| \approx\left|U_{\tau 3}\right| \approx 1 / \sqrt{2}$, and that solar-neutrino mixing should also be (close to) maximal, $\left|U_{e 1}\right| \approx\left|U_{e 2}\right| \approx 1 / \sqrt{2}$.

Now, $\left|U_{e 3}\right| \ll 1$ and maximal solar mixing are easy to explain or predict through a model. Indeed, it suffices, for instance, to assume an approximate $L_{e}-L_{\mu}-L_{\tau}$ symmetry; this has been noticed before by several authors [2, i3. atmospheric mixing is not easy to explain or predict by a model (as distinct from a "texture" or an Ansatz). This is because the mass matrix of the charged leptons is in general non-diagonal, and its diagonalization destroys the maximal mixing that may follow from the mass matrix of the neutrinos.

The proposal of Ref. [i]1] , then, is to assume that $L_{e}, L_{\mu}$, and $L_{\tau}$ are separately conserved and that they are broken only softly. It follows from this assumption that the mass matrix of the charged leptons $e, \mu$, and $\tau$ is automatically diagonal, and this opens the way for explaining maximal atmospheric mixing, like I suggested above.

The idea of soft breaking of the individual lepton numbers is implemented within the context of the seesaw mechanism 倩. Thus, we assume that there are three right-handed neutrinos, each of them carrying one unit of either $L_{e}, L_{\mu}$, or $L_{\tau}$. We moreover assumeand this is crucial - that the scalar sector of our theory only contains doublets of the weak isospin, i.e. we allow for neither triplets nor singlets. The mass terms for the six neutrinos are then of the form

$$
\frac{1}{2}\left(\nu_{L}^{T} C^{-1},-\bar{\nu}_{R}\right)\left(\begin{array}{cc}
0 & M_{D} \\
M_{D} & M_{R}
\end{array}\right)\left(\begin{array}{c}
\nu_{L} \\
C \bar{\nu}_{R}^{T}
\end{array}\right)+\text { h.c. }
$$

The zero upper-left submatrix follows from the absence of scalar triplets. The Dirac mass matrix $M_{D}$ is diagonal, while the right-handed-neutrinos Majorana mass matrix $M_{R}$ is non-diagonal. The seesaw mechanism assumes that the mass terms in $M_{R}$ are super-large, and we therefore have soft breaking of the lepton numbers through super-high mass terms! (Notice that the mass terms contained in $M_{R}$ are soft because there are no scalar singlets in our theory and $M_{R}$ therefore does not arise out of any vacuum expectation value.) Now we claim that, in this framework, all lepton-flavour-changing processes are suppressed by inverse powers of the large mass scale and/or by small Yukawa couplings. We are now performing detailed calculations in order to elucidate these suppression mechanisms 
In the context of this framework we [i.1] propose our "model one", which predicts maximal atmospheric mixing and $U_{e 3}=0$. The model contains three scalar doubletseach of them having lepton-flavour-diagonal Yukawa couplings - and two $Z_{2}$ symmetries:

$$
\begin{aligned}
& Z_{2}^{(1)}: \nu_{\mu R} \leftrightarrow \nu_{\tau R}, D_{\mu} \leftrightarrow D_{\tau}, \mu_{R} \leftrightarrow \tau_{R}, \phi_{3} \rightarrow-\phi_{3} ; \\
& Z_{2}^{(2)}: \quad \mu_{R} \rightarrow-\mu_{R}, \tau_{R} \rightarrow-\tau_{R}, \phi_{2} \rightarrow-\phi_{2}, \phi_{3} \rightarrow-\phi_{3},
\end{aligned}
$$

where $D_{\mu}$ and $D_{\tau}$ are lepton doublets, while $\mu_{R}$ and $\tau_{R}$ are charged-lepton singlets. From these two symmetries - note in particular the interchange of $\mu$ and $\tau$ indices through $Z_{2}^{(1)}$ follow neutrino mass matrices of the form

$$
M_{D}=\left(\begin{array}{ccc}
a & 0 & 0 \\
0 & b & 0 \\
0 & 0 & b
\end{array}\right), \quad M_{R}=\left(\begin{array}{ccc}
x & y & y \\
y & z & w \\
y & w & z
\end{array}\right) .
$$

It is easy to see that, then, the effective mass matrix for the light neutrinos, $-M_{D} M_{R}^{-1} M_{D}$, is of exactly the same form as $M_{R}$. That form of the effective mass matrix leads to $U_{e 3}=0$ and to maximal atmospheric mixing - to $\left|U_{\mu 3}\right|=\left|U_{\tau 3}\right|=1 / \sqrt{2}$. (This fact was recognized by a number of people before us [i] model which justifies that form of the neutrino mass matrix.) Most important, the chargedlepton mass matrix remains diagonal, and we are able to break the $\mu \leftrightarrow \tau$ symmetry and obtain $m_{\mu} \neq m_{\tau}$. The relevant Yukawa couplings are

$$
\left(\bar{D}_{\mu} \mu_{R}+\bar{D}_{\tau} \tau_{R}\right) \phi_{2} \quad \text { and } \quad\left(\bar{D}_{\mu} \mu_{R}-\bar{D}_{\tau} \tau_{R}\right) \phi_{3}
$$

The simultaneous vacuum expectation values of $\phi_{2}$ and $\phi_{3}$ allow us to obtain $m_{\mu} \neq m_{\tau}$.

In the context of the same framework, we [i] may construct "model two", independent of "model one", which leads to (almost-)maximal solar mixing. For this purpose it is enough to assume that there is an approximate $L_{e}-L_{\mu}-L_{\tau}$ symmetry in $M_{R}$. That symmetry implies

$$
\left(M_{R}\right)_{e \mu},\left(M_{R}\right)_{e \tau} \gg\left(M_{R}\right)_{e e},\left(M_{R}\right)_{\mu \mu},\left(M_{R}\right)_{\tau \tau},\left(M_{R}\right)_{\mu \tau},
$$

because $\left(M_{R}\right)_{e \mu}$ and $\left(M_{R}\right)_{e \tau}$ do not break $L_{e}-L_{\mu}-L_{\tau}$. If we denote by $\epsilon$ the order of magnitude of the ratio of the masses on the right-hand-side of eq. (i.ji $)$ over the ones on the left-hand-side, then $\epsilon$ is small and we easily obtain

$$
\begin{aligned}
\left|U_{e 3}\right| & \sim \epsilon, \\
1-\sin ^{2}\left(2 \theta_{\odot}\right) & \sim \epsilon^{2}, \\
\Delta m_{\odot}^{2} / \Delta m_{\text {atm }}^{2} & \sim \epsilon^{3} .
\end{aligned}
$$

With a suitable $\epsilon$ one may then, for instance, fit for the large-mixing-angle MSW solution of the solar-neutrino puzzle, which seems at present to be the experimentally preferred one.

I want to stress that model two is an alternative to model one: the two models may-or may not - be used together. If we use them together - by assuming model one with its three 


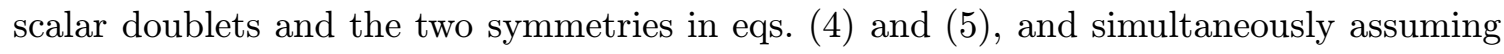
that there is an approximate $L_{e}-L_{\mu}-L_{\tau}$ symmetry in $M_{R}$ - then we shall naturally obtain bimaximal mixing. However, this is not a necessary option.

In conclusion, I have suggested that, in the context of the seesaw mechanism for suppression of the neutrino masses, the three lepton numbers $L_{e}, L_{\mu}$, and $L_{\tau}$ may be separately conserved in the Yukawa couplings while they are softly broken, at a high scale, in the Majorana mass matrix of the singlet neutrinos. I have shown that this yields a framework within which self-contained, consistent theories for maximal atmospheric-neutrino mixing and/or for maximal solar-neutrino mixing may be constructed.

\section{References}

[1] W. Grimus and L. Lavoura, Softly broken lepton numbers and maximal neutrino mixing, J. High Energy Phys. 07 (2001) 045 [hep-ph/0105212].

[2] S.T. Petcov, On pseudo-Dirac neutrinos, neutrino oscillations and neutrinoless double beta decay, Phys. Lett. B 110 (1982) 245;

C.N. Leung and S.T. Petcov, A comment on the coexistence of Dirac and Majorana massive neutrinos, Phys. Lett. B 125 (1983) 461;

R. Barbieri, L.J. Hall, D. Smith, A. Strumia and N. Weiner, Oscillations of solar and atmospheric neutrinos, J. High Energy Phys. 12 (1998) 017 [hep-ph/9807235].

[3] L. Lavoura, New model for the neutrino mass matrix, Phys. Rev. D 62 (2000) 093011 [hep-ph/0005321];

W. Grimus and L. Lavoura, A neutrino mass matrix with seesaw mechanism and two-loop mass splitting, Phys. Rev. D 62 (2000) 093012 [hep-ph/0007011];

L. Lavoura and W. Grimus, Seesaw model with softly broken $L_{e}-L_{\mu}-L_{\tau}$, J. High Energy Phys. 09 (2000) 007 [hep-ph/0008020].

[4] M. Gell-Mann, P. Ramond and R. Slansky, Complex spinors and unified theories, in Supergravity, D.Z. Freedman and F. van Nieuwenhuizen eds., North Holland, Amsterdam 1979;

T. Yanagida, in Proceedings of the workshop on unified theory and baryon number in the universe, O. Sawata and A. Sugamoto eds., KEK, Tsukuba, Japan 1979;

R.N. Mohapatra and G. Senjanović, Neutrino mass and spontaneous parity violation, Phys. Rev. Lett. 44 (1980) 912.

[5] W. Grimus and L. Lavoura, in preparation.

[6] E. Ma and M. Raidal, Neutrino mass, muon anomalous magnetic moment, and lepton flavor nonconservation, Phys. Rev. Lett. 87 (2001) 011802 [hep-ph/0102255];

K.R.S. Balaji, W. Grimus and T. Schwetz, The solar LMA neutrino oscillation solution in the Zee model, Phys. Lett. B 508 (2001) 301 [hep-ph/0104035];

C.S. Lam, A 2-3 symmetry in neutrino oscillations, Phys. Lett. B 507 (2001) 214

[hep-ph/0104116]. 